\title{
The NOAO Variable-Sky Project
}

\author{
T. Matheson, R. Blum, B. Jannuzi, T. Lauer, D. Norman, \\ K. Olsen, S. Ridgway, A. Saha, R. Shaw, and A. Walker \\ National Optical Astronomy Observatory, Tucson, AZ 85719, USA \\ email: matheson@noao.edu
}

\begin{abstract}
Modern time-domain surveys have demonstrated that finding variable objects is relatively straightforward. The problem now is one of selecting and following up discoveries. With even larger-scale surveys on the horizon, the magnitude of the problem will inevitably increase. One way to prepare for the coming deluge is to have realistic estimates of the numbers of potential detections so that resources can be developed to meet that need. To that end, astronomers at the National Optical Astronomy Observatory (NOAO) have begun a project to characterize the variable sky in terms of type of objects, distribution on the sky and range of variation.
\end{abstract}

Keywords. Surveys

\section{Introduction}

The transient alert rate from LSST will be tremendous (LSST Science Book 2009). Estimates of the number of detections per night have ranged over many orders of magnitude, and knowing the scale of the problem is critical to anticipating future observing requirements. If the science to be done with variables requires follow-up observations on rapid (or even not so rapid) time-scales, then we will need to have those facilities in place to handle the flow of alerts from LSST. That need will include photometric and spectroscopic instruments for virtually all accessible wavelengths. Having reliable estimates of the numbers of objects will make for more efficient allocations of resources to develop those facilities and observing strategies.

In our approach we include both theoretical models and empirical studies to predict the number of variables that can be detected in any given pointing of a time-domain survey. We test them against prior and on-going surveys, and also with our own on-sky experiments. The ultimate goal is an easily accessible tool that any astronomer can use to predict numbers and distributions of variables for any general time-domain project.

\section{Solar System}

A large source of transient objects in any time-domain survey is the Solar System itself. There are many classes of objects, not all confined to the ecliptic plane. The Grav et al. (2011) model of Solar System bodies contains over 14 million objects from 10 broad categories (main-belt asteroids, near-Earth objects, Trojans, Centaurs, trans-Neptunian objects, scattered disk objects, potential Earth impactors, short-period comets, longperiod comets and hyperbolic comets). From the orbital elements provided by that model we can predict the number of objects for a given field of view and their magnitude distribution for any particular pointing (see Fig. 1).

There are some subtleties involved in using this model. The epoch for which coordinates are derived is an important consideration. For Solar System objects, the relative 


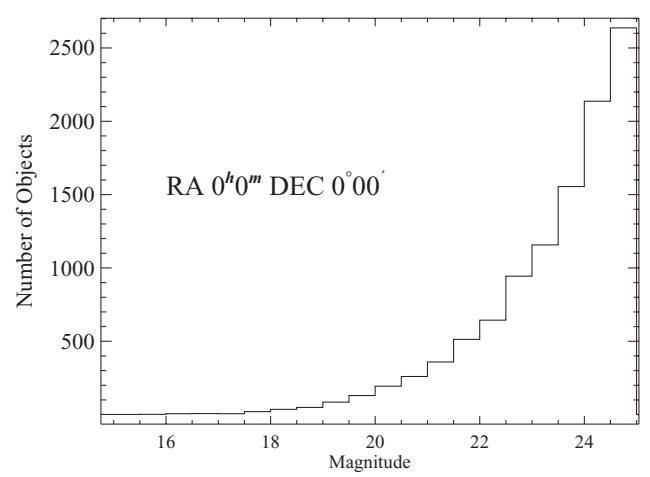

Figure 1. Magnitude distribution of the Solar System objects from the Grav model in the LSST field of view $\left(9.6 \mathrm{deg}^{2}\right)$ pointed at the LSST reference field $\left(l=96^{\circ}, \mathrm{b}=-60^{\circ}\right.$, which is also RA $0^{h}$, Dec $0^{\circ}$ and in the ecliptic plane). There are 10752 objects brighter than $V=25$ (the approximate depth of single-epoch LSST images). If we go off the ecliptic by $23^{\circ}$, there are 926 objects.

positions of the Sun, Earth and the individual body can dramatically affect the visibility of the body. This includes not only the relative distance of the main belt from the Earth (depending on whether you are looking at opposition or conjunction), but also the illumination of the surface visible from Earth. In addition, the orbital solutions do not include perturbations, but for the purposes of this exercise the position of any individual object is not as important as the overall number of expected objects in the given field. Finally, any model has limitations in completeness, but missing elements will in general be rare.

\section{Galactic Variables}

To estimate the number of Galactic variables, we first constructed a simulation of the stellar content in a given field of view using the Besançon Galaxy model (Robin et al. 2003). This model generates stars based on the Galactic components in the field of view. We plan to explore other Galactic models as well. We then selected only those stars that would be detected in a single-epoch visit by LSST. As we know the basic parameters of each star, we can determine which stars are in the instability strip (e.g., Gautschy \& Saio 1995) and may thus appear as variable. In the LSST reference field (see Fig. 2) there are $\sim 100$ instability-strip variables. Other types of stellar variables will require further analysis.

A different approach is to look at variability by stellar type as derived from previous studies. We used the results from Kepler (Ciardi et al. 2011) and HATNet (Hartman et al. 2011) to predict the total number of stellar variables based on their empirical calibration. Here we define variability as changing enough to meet the $5 \sigma$ level of LSST. Combining the probability of variation with the number of stars of that class in magnitude bins enables us to derive the numbers of expected variables. For the LSST reference field, that total is $\sim 800$ stellar variables. This technique has some limitations (especially long-period variability because of the duration of the Kepler mission), but it does provide a realistic estimate for those variables that were accessible in the prior studies. 


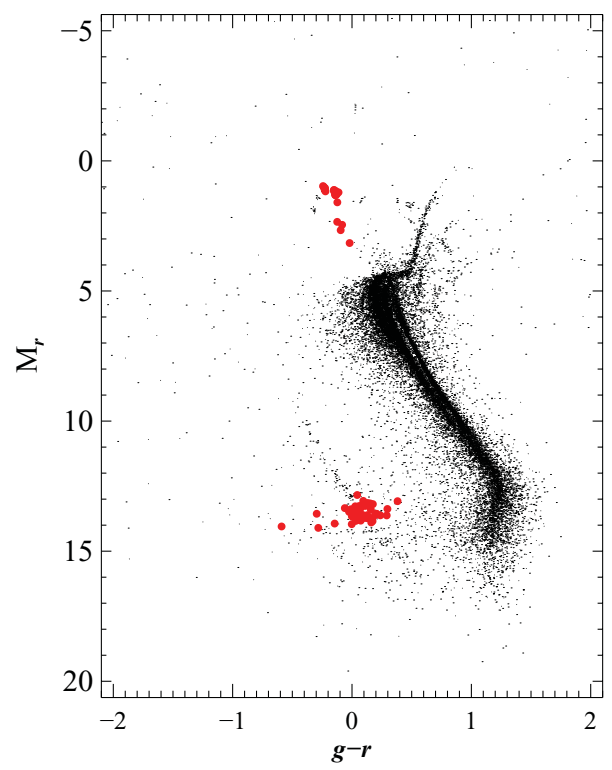

Figure 2. Colour-magnitude diagram of the stars generated from the Besançon Galaxy model for a $10-\mathrm{deg}^{2}$ field of view at the LSST reference field $\left(l=96^{\circ} \mathrm{b}=-60,{ }^{\circ} \mathrm{RA} 0^{h}\right.$, Dec $\left.0^{\circ}\right)$. Note that the nominal LSST FOV is $9.6 \mathrm{deg}^{2}$. There are 105982 stars brighter than $r=25$, and 93 instability-strip variables (large dots). Most of the instability-strip variables are pulsating white dwarfs.

\section{Future Work}

Although this paper focusses on results applicable to LSST, the techniques can be used for any time-domain survey. Given the field of view and depth of each single-epoch visit, the same results can be generated to match other surveys.

This is work in progress. The next steps will be to check further the results described here, both with models and on-sky calibrations. We will explore models to predict stellar variables outside the instability strip, in order to tie together theoretical predictions with the empirical variability results. We will incorporate models of local galaxy distributions to accommodate stellar variables detectable in nearby galaxies. We will also add the next major component - extragalactic variables. Finally, for this programme to be of general use we will develop an interface that allows users to enter their own survey parameters and thence to obtain realistic predictions of expected numbers of variables.

\section{References}

Ciardi, D. R., von Braun, K., Bryden, G., van Eyken, J., Howell, S. B., Kane, S. R., Plavchan, P., Ramirez, S. V., \& Stauffer, J. R. 2011, AJ, 141, 108

Gautschy, A. \& Saio, H. 1995, ARAA, 33, 75

Grav, T., Jedicke, R., Denneau, L., Chesley, S., Holman, M. J., \& Spahr, T. B. 2011, PASP, 123,423

Hartman, J. D., Bakos, G. A., Noyes, R. W., Sipocz, B., Kovacs, G., Mazeh, T., Shporer, A., \& Pal, A. 2011, $A J, 141,166$

LSST Science Collaborations \& LSST Project 2009, LSST Science Book, Version 2.0, arXiv:0912.0201, http://www.lsst.org/lsst/scibook

Robin, A. C., Reyle, C., Derriere, S., \& Picaud, S. 2003, A\&A, 409, 523 